\title{
Optical information processing in Bose-Einstein condensates
}

\author{
LENE VESTERGAARD HAU
}

is at the Lyman Laboratory, Harvard University, Cambridge, Massachusetts 02138, USA.

e-mail: hau@physics.harvard.edu

\section{Slow-light effects in ultracold atom clouds known as Bose-Einstein condensates offer rich opportunities for storing and processing optical signals.}

A lthough the high speed of light, $300,000 \mathrm{~km} \mathrm{~s}^{-1}$ in a vacuum, makes it possible to transmit information with almost no delay, even over ultralong distances, there are strong motivations for using the ability to dramatically slow light down. In particular, the creation of more efficient and sophisticated optical communication networks in the future will probably require direct control over the speed at which optical information propagates. For instance, a key ingredient of such networks will be the all-optical router - a device that controls the flow and timing of optical data without the need to convert them to electronic signals. Such all-optical routers will require dynamically controllable optical delays and the ability to perform short-term storage of light pulses.

The capability of controlling light in this manner may be just the beginning. It could ultimately lead to more advanced forms of optical processing where the actual information content sent across the network is determined by conditional processing of the optical inputs from many distant nodes in the network.

When considering information processing, an attractive characteristic of laser light is that of phase coherence, meaning that information may be encoded in both the amplitude and phase of the electromagnetic wave. For optical processing applications it is thus attractive to find a medium that can preserve this phase coherence, and slow light in Bose-Einstein condensed atomic gases provides this unique ability.

In recent years, it has been observed that light can be slowed by factors of

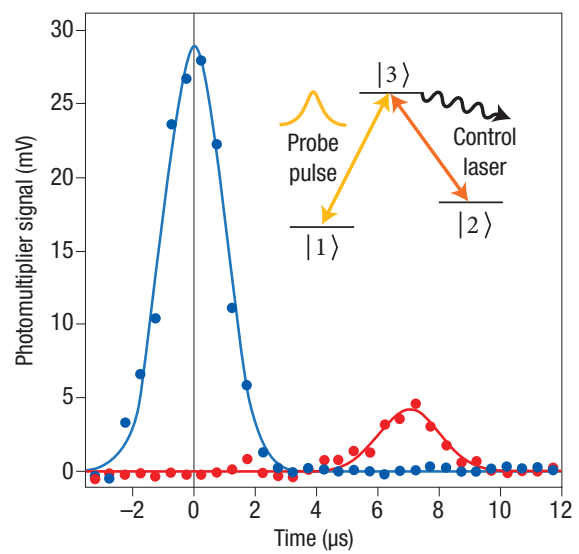

Figure 1 Light pulse (red) slowed in a 200-micrometre-long atom cloud cooled below one microkelvin (ref. 1). In this experiment, it takes the pulse 7 microseconds to traverse the cloud, and light is slowed by a factor of ten million. The reference pulse (blue) is recorded with no atoms. The curves represent Gaussian fits to the measured pulses. The insert shows the three internal atomic levels coupled by the 'probe' laser pulse and the 'control' (reference) laser.

tens to hundreds of millions in clouds of atoms, as shown in Fig. 1 (ref. 1). This phenomenon is achieved by manipulating the internal structure of the atoms with two laser fields: a 'control' (reference) laser beam and a typically weaker, pulsed 'probe' laser beam. The control laser, which is resonant with the internal $|2\rangle \rightarrow|3\rangle$ transition of an atom (insert in Fig. 1), illuminates atoms that are initially in state $|1\rangle$. Owing to quantum interference effects, the presence of the control beam allows a probe laser pulse with a centre frequency tuned to the $|1\rangle \rightarrow|3\rangle$ resonance to be slowed and transmitted through the cloud with minimal loss.

In the process, the probe and control laser fields adiabatically change the internal atomic state into a quantummechanical 'dark' superposition state $|D\rangle$ where the atom is partly in $|1\rangle$ and at the same time partly in $|2\rangle$. More precisely, the population amplitude of state $|2\rangle, c_{2}$, relative to that of state $|1\rangle, c_{1}$, is proportional to the ratio of the electricfield amplitudes of the probe, $E_{\mathrm{p}}$, and control laser fields, $E_{c}$ :

$$
\begin{gathered}
|D\rangle=c_{1}|1\rangle+c_{2}|2\rangle ; \\
\frac{c_{2}}{c_{1}} \propto-\frac{E_{\mathrm{p}}}{E_{\mathrm{c}}} .
\end{gathered}
$$

Absorption of a probe photon would lead to excitation of the atom from $|1\rangle$ to $|3\rangle$. However, this final state $|3\rangle$ can also be reached by another path, namely by the atom's absorption of a control laser photon and associated excitation from $|2\rangle$ to $|3\rangle$. For an atom in the dark state, the transition amplitudes associated with these two paths destructively interfere, resulting in no loss (absorption) from either laser field.

During formation of the dark state, the atomic population amplitude is transferred from state $|1\rangle$ to state $|2\rangle$ by probe photon absorption and stimulated emission into the control laser field, securing coherence of the process. Perfect destructive quantum interference is present only when the probe laser is exactly resonant with the $|1\rangle \rightarrow|3\rangle$ transition. This leads to a narrow 

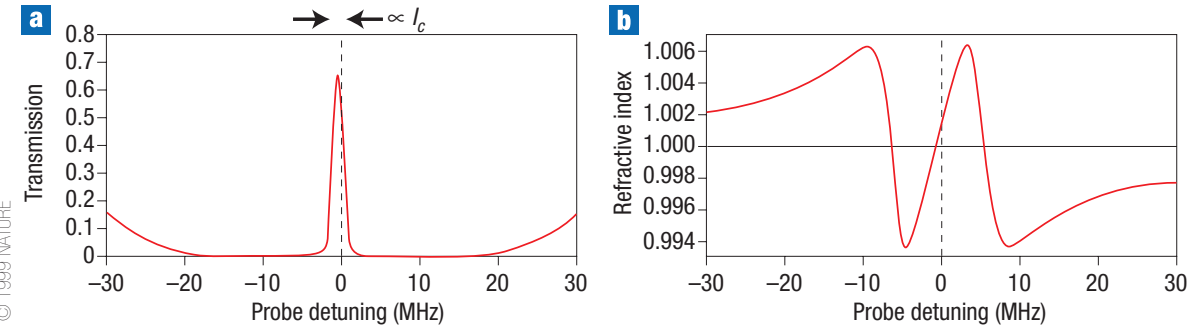

Figure 2 Slow-light profiles ${ }^{1}$. a, The transmission and, b, refractive-index profile for slow light. The group (signal) velocity of a light pulse is inversely proportional to the slope of the refractive-index curve around resonance, and can be controlled dynamically by $I_{c}$. The transmission bandwidth is also proportional to this intensity. The two laser fields transfer atoms into 'dark' superposition states $(|D\rangle)$ whereby optical amplitude and phase information is imprinted on the atoms.

transmission window centred at the resonance frequency, and also to a very rapid variation of the refractive index, $n$, with frequency, $\omega$, for the probe laser field (see ref. 2 and Fig. 2). The group velocity of a light pulse, $v_{\mathrm{g}}$, is inversely proportional to the slope, $\mathrm{d} n / \mathrm{d} \omega$, and is controlled by the intensity of the control laser, $I_{c}$ :

$$
v_{\mathrm{g}}=\frac{c}{n+\omega \frac{\mathrm{d} n}{\mathrm{~d} \omega}} \propto I_{c},
$$

where $c$ is the vacuum velocity of light.

With cold atoms, Doppler shifts in frequency are minimized, and for very low control-laser intensity, light speeds of just a few metres per second can be obtained even for counter-propagating control and probe laser fields. During the slowing down process, the light pulse is spatially compressed. For typical experimental parameters, it can be compressed from 1 kilometre to only 20 micrometres. Through dark-state formation, an imprint of the probe light pulse is mapped onto the atom cloud, and the imprint contains both the amplitude (shape) and phase information of the pulse. This holographic imprint follows the light pulse as it slowly propagates through the atom cloud. On exit of the cloud, the light pulse speeds back up to the usual (very high) light speed, stretching back out and regaining its initial length.

By blocking the control laser beam when a co-propagating probe light pulse has slowed down and is compressed enough to be contained within the atom cloud, this light pulse can be halted and extinguished, while the atomic hologram stays in the cloud. When the control laser is turned on again, the process is reversed; the probe light pulse is regenerated and moves on. Such storage of optical information has been demonstrated for classical light pulses containing many photons $\mathrm{s}^{3,4}$, for singlephoton light pulses ${ }^{5,6}$, and for entangled light fields ${ }^{7}$.

Instead of storing just a single light pulse in an atom cloud, a threedimensional image may also be injected, slowed and stored. During the slowlight process, the image information is compressed in the direction of propagation. Two- and three-dimensional images have indeed been slowed and stored in a warm alkali gas ${ }^{8,9}$. In these experiments, the optical phase parameter was used to compensate for the thermal smearing of the stored patterns, that result from atomic motion in the hot gas during the storage time. With the use of cold atoms, such thermal smearing can be minimized ${ }^{3}$.

It is particularly interesting to use ultracold atom clouds, in the form of Bose-Einstein condensates ${ }^{10,11}$, for storing information. A Bose-Einstein condensate is fundamentally different from even very cold, non-condensed clouds. Bose-Einstein condensates are phase-coherent objects, and changes of the stored holographic imprint owing to atom dynamics do not lead to loss clouds; rather, they lead to processing. As very rich nonlinear dynamics can occur in superfluid Bose-Einstein condensates, and as the dynamics can easily be controlled by controlling atomatom interactions (for example, with external magnetic fields), Bose-Einstein condensates can be very powerful processors of optical information.

The potential for using Bose-Einstein condensates as optical processors is illustrated by recent experiments with double condensates ${ }^{12}$. In these experiments, the first condensate is illuminated with a control laser field, and a counter-propagating probe light of information as in non-condensed

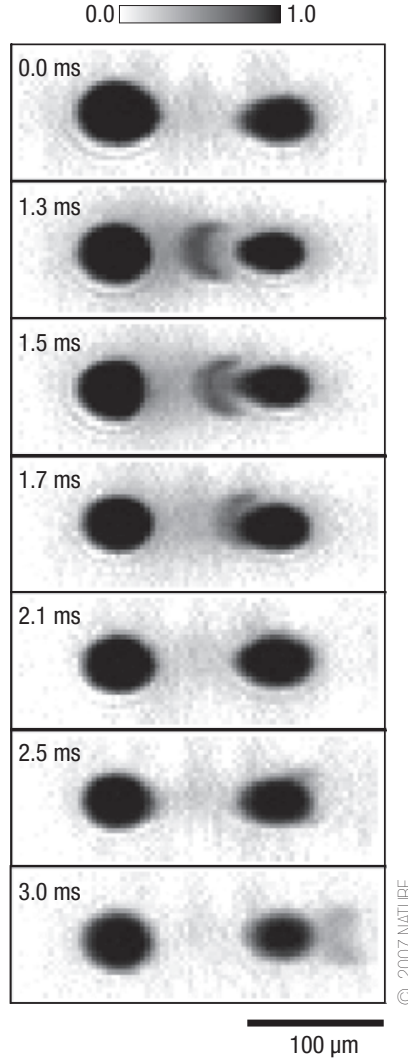

Figure 3 Flying matter copy ${ }^{12}$. The data carried by a light pulse stopped and extinguished in the first (left) Bose-Einstein condensate are converted onto a matter copy that travels across to the second (right) Bose-Einstein condensate, and then converted back to light. In the experimental images, the boomerang shaped matter copy is seen moving between the two Bose-Einstein condensates, and it contains the same information as the extinguished light pulse: it has the same shape and holds the same phase information.

pulse is injected, slowed and compressed, and then stopped and extinguished by blocking the control laser. For this counter-propagating geometry, the atomic hologram receives a two-photon recoil kick and starts moving at a speed of 200 metres per hour - it exits the first condensate and moves into free space. Thereby, a perfect matter copy of the probe light pulse, which has already been extinguished in the first condensate, now exists in free space. The matter copy (a pulse of atoms) contains the same information as the original probe light pulse - it has the same shape and contains the same phase information.

The imprint can be photographed and can be seen to move freely in space (Fig. 3). Eventually it reaches the second condensate and once it is imbedded, the matter copy can be 

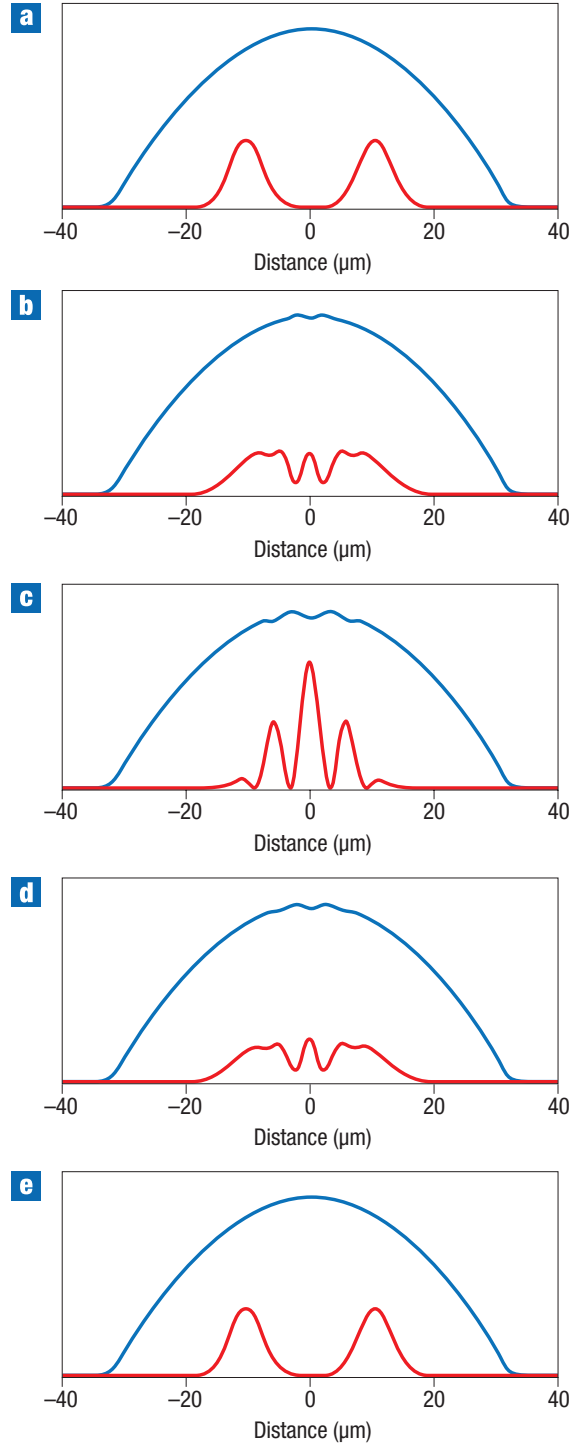

Figure 4 Two-gate conditional processing. Two optical input pulses are slowed and stored in a Bose-Einstein condensate of $|1\rangle$ atoms with a density profile as shown by the blue curve. a, State $|2\rangle$ atomic imprints (red) are generated. b-d, The subsequent two-component condensate dynamics (calculation by Zachary Dutton; see also ref. 14). Note that when the state $|2\rangle$ imprints overlap, interference fringes are created. e, After a time interval (in the millisecond regime for typical experimental parameters), the initial state $|2\rangle$ amplitude patterns are regenerated but their phases have changed. This processing is similar to that of a two-cell automaton. converted back to light - the probe light pulse is regenerated in the second condensate by simply unblocking the control laser. To achieve this, it is of paramount importance that BoseEinstein condensates are used. When the probe light pulse is recreated, the atoms in the travelling-matter copy act as little radiating antennas; it is the phase-coherent nature of the receiving condensate that phase-locks these antennas and allows the probe light pulse to be coherently regenerated.

In these experiments, light is converted to matter and then back to light. Once it is in matter form, the pulse is easily manipulated - 'sculpted' by laser fields for example - and when it is subsequently converted back to light, the changes induced on the matter copy are present in the regenerated light field. Such manipulation represents a particularly powerful way of storing and processing optical information. Optical delays with durations equal to 10,000 pulse widths have been obtained in this system for input probe light pulses $200 \mathrm{~ns}$ long ${ }^{12}$. The bandwidth of the system can be manipulated and is directly proportional to the intensity of the control laser.

It is interesting to speculate regarding the further possibilities of slow light for processing applications. One intriguing example could be processing similar to that of a cellular automaton ${ }^{13}$. A one-, two- or three-dimensional pattern of input probe light pulses would be stored in a condensate and form a pattern of 'cells'. A very simple example (a two-cell system) is shown in Fig. 4. Two light pulses are slowed and stored in a BoseEinstein condensate and accordingly, two imprints of state $|2\rangle$ atomic amplitude are formed in the condensate of state $|1\rangle$ atoms (Fig. 4a).

Coherent dynamics of the resulting two-component condensate controls the subsequent processing (Fig. $4 \mathrm{~b}-\mathrm{d}$ ). After a certain time step, the state $|2\rangle$ amplitude patterns are regenerated (Fig. 4e), but the phases of the imprints have changed, with the phase change of each imprint depending on the presence of the other imprint. This system could be expanded to form a larger network of cells where the dynamics of one particular cell would depend on the information content in neighbouring cells. (Such processing could also be performed in the quantum regime by using singlephoton or entangled input pulses, for example). After a discrete time step, the output pattern forming the cells of the 'next generation' would be determined by the input pulse pattern - cells of the preceding generation - and by the global dynamics of the condensate. The global dynamics are determined by atom-atom interactions that can easily be controlled as mentioned above.

So far we have only seen the 'tip of the iceberg' in terms of using slow light for optical control, and prospects for high-level processing are very intriguing and promising. With Bose-Einstein condensates, the phase coherence of the processor is matched to the phase coherence of the ideal information carrier (laser light), whereas the slowlight process enables perfect information exchange between these two systems. If we can meet the challenge of creating slow light and Bose-Einstein condensates in a 'practical system' (and why not), we would certainly obtain unprecedented information processing capabilities.

\section{References}

1. Hau, L. V., Harris, S. E., Dutton, Z. \& Behroozi, C. H. Nature 397, 594-598 (1999).

2. Harris, S. E., Field, J. E. \& Kasapi, A. Phys. Rev. A 46, R29-R32 (1992).

3. Liu, C., Dutton, Z., Behroozi, C. H. \& Hau, L. V. Nature 409, 490-493 (2001).

4. Phillips, D. F., Fleischhauer, A., Mair, A., Walsworth, R. L. \& Lukin, M. D. Phys. Rev. Lett. 86, 783-786 (2001).

5. Chaneliere, T. et al. Nature 438, 833-836 (2005).

6. Eisaman, M. D. et al. Nature 438, 837-841 (2005).

7. Choi, K. S., Deng, H., Laurat, J. \& Kimble, H. J. Nature 452, 67-71 (2008).

8. Pugatch, R., Shuker, M., Firstenberg, O., Ron, A. \& Davidson, N Phys. Rev. Lett. 98, 203601 (2007).

9. Vudyaseto, P. K., Camacho, R. M. \& Howell, J. C. Phys. Rev. Lett. 100, 123903 (2008).

10. Cornell, E. A. \& Wieman, C. E. Rev. Mod. Phys. 74, 875-893 (2002).

11. Ketterle, W. Rev. Mod. Phys. 74, 1131-1151 (2002)

12. Ginsberg, N. S., Garner, S. R. \& Hau, L. V. Nature 445, 623-626 (2007).

13. Wolfram, S. A New Kind of Science (Wolfram Media, Champaign, Illinois, 2002).

14. Dutton, Z. \& Hau, L. V. Phys. Rev. A 70, 053831 (2004). 